\title{
AHLFORS-BEURLING CONFORMAL INVARIANT AND RELATIVE CAPACITY OF COMPACT SETS
}

\author{
VLADIMIR N. DUBININ AND MATTI VUORINEN
}

(Communicated by Jeremy Tyson)

\begin{abstract}
For a given domain $D$ in the extended complex plane $\overline{\mathbb{C}}$ with an accessible boundary point $z_{0} \in \partial D$ and for a subset $E \subset D$, relatively closed w.r.t. $D$, we define the relative capacity relcap $E$ as a coefficient in the asymptotic expansion of the Ahlfors-Beurling conformal invariant $r(D \backslash E, z) / r(D, z)$ when $z$ approaches the point $z_{0}$. Here $r(G, z)$ denotes the inner radius at $z$ of the connected component of the set $G$ containing the point $z$. The asymptotic behavior of this quotient is established. Further, it is shown that in the case when the domain $D$ is the upper half plane and $z_{0}=\infty$, the capacity relcap $E$ coincides with the well-known half-plane capacity hcap $E$. Some properties of the relative capacity are proven, including the behavior of this capacity under various forms of symmetrization and under some other geometric transformations. Some applications to bounded holomorphic functions of the unit disk are given.
\end{abstract}

\section{INTRODUCTION}

Let $D_{1}$ and $D_{2}$ be domains having Green functions in the extended complex plane $\overline{\mathbb{C}}$, and let the point $z \in D_{1} \subset D_{2}$. We denote by $r\left(D_{k}, z\right)$ the inner radius of the domain $D_{k}, k=1,2$, at the point $z$ (see e.g. [H], [D1]). The quotient $r\left(D_{1}, z\right) / r\left(D_{2}, z\right)$ is conformal invariant in the sense that for every conformal map $f$ of $D_{2}$ we have the equality

$$
\frac{r\left(D_{1}, z\right)}{r\left(D_{2}, z\right)}=\frac{r\left(f\left(D_{1}\right), f(z)\right)}{r\left(f\left(D_{2}\right), f(z)\right)} .
$$

The study of this kind of invariant expression goes back to the works of Ahlfors and Beurling [A, p. 436]. Some significant applications to geometric theory of functions are given in $\mathrm{A}, \mathrm{AB}, \mathrm{He}, \mathrm{Pom}$. In this paper we study the behavior of the invariant $r\left(D_{1}, z\right) / r\left(D_{2}, z\right)$ when the point $z$ tends to a given common boundary point of the domains $D_{1}$ and $D_{2}$. More precisely, we investigate the following situation. Let $D$ be a domain in $\overline{\mathbb{C}}$, and let $z_{0}$ be an accessible boundary point of the domain $D$. Suppose that in a neighborhood (with respect to $D$ ) of the point $z_{0}$ the boundary

Received by the editors March 10, 2012 and, in revised form, September 10, 2012; September 27, 2012; and November 30, 2012.

2010 Mathematics Subject Classification. Primary 30C85.

Key words and phrases. Conformal invariant, inner radius, holomorphic function, Schwarzian derivative.

The research of the first author was supported by the Russian Foundation for Basic Research, project 11-01-00038.

The research of the second author was supported by the Academy of Finland, project 2600066611 . 
$\partial D$ is represented by an analytic arc $\gamma$ (in the case $z_{0}=\infty$ it is required that the image of the arc $\gamma$ under the mapping $z \mapsto 1 / z$ be analytic). Consider an arbitrary set $E \subset D$, relative closed with respect to $D$ such that the inner distance $\rho\left(E, z_{0}\right)$ from the point $z_{0}$ to the set $E$ with respect to the domain $D$ is positive. In the case of a finite point $z_{0}$, the relative capacity relcap $E$ of the set $E$ is defined via the following asymptotic expansion:

$$
\frac{r(D \backslash E, z)}{r(D, z)}=1-2(\operatorname{relcap} E)\left|z-z_{0}\right|^{2}+o\left(\left|z-z_{0}\right|^{2}\right), \quad z \rightarrow z_{0},
$$

where the approach of $z$ to the point $z_{0}$ takes place along an arbitrary arc in $D$ perpendicular to $\gamma$ at the point $z_{0}$. Here $r(G, z)$ denotes the inner radius of the connected component of the set $G$ containing the point $z$. If the point $z_{0}=\infty$, then the parameter $\left|z-z_{0}\right|$ in the definition of the relative capacity relcap $E$ is replaced by $|1 / z|$. We say that relcap $E$ is the relative capacity of the set $E$ with respect to the domain $D$ at the point $z_{0}$. We shall establish the asymptotic expansion (1.1) in Section 2. It will also be shown that in the case when the domain $D$ is the upper half plane $H$ and the point $z_{0}=\infty$ we have

$$
\text { relcap } E=\text { hcap } E \text {, }
$$

where hcap $E$ is the half-plane capacity of the set $E$ [L1]. In the case when the set $H \backslash E$ is a simply-connected domain, the equality of the relative and halfplane capacities was proven in [D2, with essential use of conformal mapping (cf. [D2, formula (3)]). We recall further that the notion of half-plane capacity arises naturally in statistical physics when examining the Schramm-Löwner equations (see G. Lawler [L1, L2, L3]). There are several definitions of this capacity in the literature. For example, let $G=H \backslash E$ and

$$
\phi_{G}(z)=\operatorname{Im} z-\mathbf{E}^{z}\left[\operatorname{Im}\left(B_{\tau_{G}}\right)\right],
$$

where $B_{t}$ is a standard Brownian motion and $\tau_{G}=\inf \left\{t: B_{t} \notin G\right\}$, and $\mathbf{E}^{z}$ is the mathematical expectation. Then $\phi_{G}$ is a positive harmonic function on $G$ that vanishes at the regular points of the boundary $\partial G$ and such that

$$
\phi_{G}(z)=\operatorname{Im} z+O\left(|z|^{-1}\right), \quad z \rightarrow \infty .
$$

The half-plane capacity (at infinity) of $E$ is defined by

$$
\phi_{G}(z)=\operatorname{Im}\left(z+\frac{\operatorname{hcap} E}{z}\right)+o\left(|z|^{-1}\right), \quad z \rightarrow \infty
$$

(see [L2, Lecture 2]). Some new geometric properties of the half-plane capacity were proved in the recent paper [D2]. Following this paper we shall examine the properties of the relative capacity relcap $E$ under various geometric transformations of the set $E$ in the case when the domain $D$ is the disk and the point $z_{0}=1$ (Section 3 ). Our results do not reduce to the corresponding statements of [D2] in the particular case $D=H, z_{0}=\infty$, because the symmetrization procedures applied here are not invariant under Möbius transformations. Finally, in Section 4 there are given some applications of the introduced capacity to holomorphic functions. The results obtained here turn out to be effective in the study of a boundary version of the Schwarz lemma involving the Schwarzian derivative (see, e.g. [S, TV, D3]). After the completion of the writing of this paper, we learned about a very recent paper of S. Rohde and C. Wong, also studying half-plane capacity [RW]. 


\section{EXISTENCE AND PROPERTIES OF RELATIVE CAPACITY}

Following the proof of Lemma 1 in the paper D2] we first establish the existence of the asymptotic expansion (1.1).

Theorem 2.1. Let $D$ be a domain in the extended complex plane $\overline{\mathbb{C}}$, and let $z_{0}$ be a finite accessible boundary point of the domain D. Suppose that in some neighborhood of $z_{0}$ the boundary $\partial D$ is represented by an analytic arc $\gamma$. Then for every set $E$ relatively closed with respect to $D$ and with $\rho\left(E, z_{0}\right)>0$, the following expansion holds:

$$
\frac{r(D \backslash E, z)}{r(D, z)}=1-c\left|z-z_{0}\right|^{2}+o\left(\left|z-z_{0}\right|^{2}\right), \quad z \in D \backslash E, z \rightarrow z_{0},
$$

where the convergence of $z$ to the point $z_{0}$ takes place along a path, perpendicular to the arc $\gamma$ at $z_{0}$ and where $c \geq 0$ is a constant, depending only on the set $E$, the domain $D$, and on the point $z_{0}$.

Proof. We consider the function $f$ mapping $D$ conformally and univalently onto a domain $f(D)$ lying in the upper half plane $H$. Extending $f$ to the arc $\gamma$ in the sense of the boundary correspondence we may assume that $f(\gamma)$ is a finite interval of the real axis and that $f\left(z_{0}\right)=0,\left|f^{\prime}\left(z_{0}\right)\right|=1$. Let $g(w, \zeta)$ be the Green function of the connected component of the symmetric set

$$
G=\{w: w \in f(\gamma) \cup f(D) \backslash f(E) \quad \text { or } \quad \bar{w} \in f(D) \backslash f(E)\}
$$

that contains the origin and let $h(w, \zeta)=\log |w-\zeta|+g(w, \zeta)$ be the regular part of this function. By the symmetry of $G$, we have

$$
g_{f(D) \backslash f(E)}(w, f(z)) \equiv g(w, f(z))-g(w, \overline{f(z)}) .
$$

Adding $\log |w-f(z)|$ to both sides and letting $w \rightarrow f(z)$, this relation gives

$$
\log r(f(D) \backslash f(E), f(z))=\log r(G, f(z))-g(f(z), \overline{f(z)}),
$$

and hence

$$
\begin{gathered}
r(f(D) \backslash f(E), f(z))=r(G, f(z)) \exp \{-g(f(z), \overline{f(z)})\} \\
=\{r(G, f(z)) r(G, \overline{f(z)}) \exp \{-2 g(f(z), \overline{f(z)})\}\}^{1 / 2} \\
=\exp \left\{\frac{1}{2}[h(f(z), f(z))+h(\overline{f(z)}, \overline{f(z)})-2 h(f(z), \overline{f(z)})+2 \log |f(z)-\overline{f(z)}|]\right\} .
\end{gathered}
$$

We consider the function $h(w, \zeta)$ as a function of four real arguments $h(u, v, \xi, \eta)$ and introduce the notation $f(z)=\Delta u+i \Delta v$. It is clear that when $z \rightarrow z_{0}$ along an arc perpendicular to $\gamma$, we have $\Delta v \rightarrow 0$ and $\Delta u=o(\Delta v), \Delta v \rightarrow 0$. The symmetric difference enclosed within the square brackets in (2.3) has the following form in the new notation:

$$
h(\Delta u, \Delta v, \Delta u, \Delta v)+h(\Delta u,-\Delta v, \Delta u,-\Delta v)-2 h(\Delta u, \Delta v, \Delta u,-\Delta v) .
$$

In view of the symmetry of the set $G$ with respect to the real axis we see that

$$
h(u, v, \xi, \eta)=h(u,-v, \xi,-\eta) .
$$

Therefore at the point $(0,0,0,0)$,

$$
\frac{\partial h}{\partial v}=\frac{\partial h}{\partial \eta}=0 .
$$


Applying this fact and applying Taylor's formula in a neighborhood of the point $(0,0,0,0)$, we have

$$
\begin{gathered}
h(\Delta u, \Delta v, \Delta u, \Delta v)-h(0,0,0,0)=\frac{\partial h}{\partial u} \Delta u+\frac{\partial h}{\partial \xi} \Delta u+\frac{1}{2}\left[\frac{\partial^{2} h}{\partial u^{2}}(\Delta u)^{2}\right. \\
+\frac{\partial^{2} h}{\partial v^{2}}(\Delta v)^{2}+\frac{\partial^{2} h}{\partial \xi^{2}}(\Delta u)^{2}+\frac{\partial^{2} h}{\partial \eta^{2}}(\Delta v)^{2}+2\left(\frac{\partial h^{2}}{\partial u \partial v} \Delta u \Delta v+\frac{\partial^{2} h}{\partial u \partial \xi}(\Delta u)^{2}\right. \\
\left.\left.+\frac{\partial^{2} h}{\partial u \partial \eta} \Delta u \Delta v+\frac{\partial^{2} h}{\partial u \partial \xi} \Delta u \Delta v+\frac{\partial^{2} h}{\partial v \partial \eta}(\Delta v)^{2}+\frac{\partial^{2} h}{\partial \xi \partial \eta} \Delta u \Delta v\right)\right]+o\left((\Delta v)^{2}\right), \Delta v \rightarrow 0, \\
h(\Delta u,-\Delta v, \Delta u,-\Delta v)-h(0,0,0,0)=\frac{\partial h}{\partial u} \Delta u+\frac{\partial h}{\partial \xi} \Delta u+\frac{1}{2}\left[\frac{\partial^{2} h}{\partial u^{2}}(\Delta u)^{2}\right. \\
+\frac{\partial^{2} h}{\partial v^{2}}(\Delta v)^{2}+\frac{\partial^{2} h}{\partial \xi^{2}}(\Delta u)^{2}+\frac{\partial^{2} h}{\partial \eta^{2}}(\Delta v)^{2}+2\left(-\frac{\partial^{2} h}{\partial u \partial v} \Delta u \Delta v+\frac{\partial^{2} h}{\partial u \partial \xi}(\Delta u)^{2}\right. \\
\left.\left.-\frac{\partial^{2} h}{\partial u \partial \eta} \Delta u \Delta v-\frac{\partial^{2} h}{\partial u \partial \xi} \Delta u \Delta v+\frac{\partial^{2} h}{\partial v \partial \eta}(\Delta v)^{2}-\frac{\partial^{2} h}{\partial \xi \partial \eta} \Delta u \Delta v\right)\right]+o\left((\Delta v)^{2}\right), \Delta v \rightarrow 0, \\
h(\Delta u, \Delta v, \Delta u,-\Delta v)-h(0,0,0,0)=\frac{\partial h}{\partial u} \Delta u+\frac{\partial h}{\partial \xi} \Delta u+\frac{1}{2}\left[\frac{\partial^{2} h}{\partial u^{2}}(\Delta u)^{2}\right. \\
+\frac{\partial^{2} h}{\partial v^{2}}(\Delta v)^{2}+\frac{\partial^{2} h}{\partial \xi^{2}}(\Delta u)^{2}+\frac{\partial^{2} h}{\partial \eta^{2}}(\Delta v)^{2}+2\left(\frac{\partial h^{2}}{\partial u \partial v} \Delta u \Delta v+\frac{\partial^{2} h}{\partial u \partial \xi}(\Delta u)^{2}-\frac{\partial^{2} h}{\partial u \partial \eta} \Delta u \Delta v\right. \\
\left.\left.+\frac{\partial^{2} h}{\partial u \partial \xi} \Delta u \Delta v-\frac{\partial^{2} h}{\partial v \partial \eta}(\Delta v)^{2}-\frac{\partial^{2} h}{\partial \xi \partial \eta} \Delta u \Delta v\right)\right]+o\left((\Delta v)^{2}\right), \quad \Delta v \rightarrow 0 .
\end{gathered}
$$

Taking into account the expression in (2.4) and substituting into (2.3) we arrive at the identity

$$
\begin{gathered}
r(f(D) \backslash f(E), f(z))=|2 \Delta v| \exp \left\{2 \frac{\partial^{2} h}{\partial v \partial \eta}(\Delta v)^{2}+o\left((\Delta v)^{2}\right)\right\} \\
=2 \Delta v+c_{1}(\Delta v)^{3}+o\left((\Delta v)^{3}\right), \quad \Delta v \rightarrow 0 .
\end{gathered}
$$

Here the constant

$$
c_{1}=4 \frac{\partial^{2} h}{\partial v \partial \eta}
$$

does not depend on the choice of the arc $\gamma$ because the left hand side is independent of this arc.

Repeating the preceding argument and replacing $f(D) \backslash f(E)$ with $f(D)$ we arrive at a similar relation:

$$
r(f(D), f(z))=2 \Delta v+c_{2}(\Delta v)^{2}+o\left((\Delta v)^{3}\right), \quad \Delta v \rightarrow 0 .
$$

Therefore

$$
\begin{aligned}
\frac{r(D \backslash E, z)}{r(D, z)}= & \frac{r(f(D) \backslash f(E), f(z))}{r(f(D), f(z))}=1+\frac{1}{2}\left(c_{1}-c_{2}\right)(\Delta v)^{2}+o\left((\Delta v)^{2}\right) \\
& =1-c\left|z-z_{0}\right|^{2}+o\left(\left|z-z_{0}\right|^{2}\right), \quad z \rightarrow z_{0} .
\end{aligned}
$$

Because the expression on the left hand side does not depend on the choice of the function $f$ and does not exceed 1 , we see that the constant $c:=-\frac{1}{2}\left(c_{1}-c_{2}\right) \geq 0$ is independent of $f$. The theorem is proved. 
The asymptotic expansion in the case $z_{0}=\infty$ is contained in what was proved above with the change of variable $z-z_{0} \mapsto 1 / z$. Note that we did not only prove the existence of the expansion (1.1), but also established a representation of the relative capacity in terms of the Green functions of the domains in question.

Remark 2.5. From the above proof it is clear that the requirement of the analyticity of the arc $\gamma$ can be weakened. It is enough to require that the arc $\gamma$ have a tangent at the point $z_{0}$, the function $f$ has an extension to the $\operatorname{arc} \gamma$ and the following expansion holds:

$$
f(z)-f\left(z_{0}\right)=a\left(z-z_{0}\right)+o\left(\left(z-z_{0}\right)\right), \quad z \in D \cup \gamma, \quad z \rightarrow z_{0},
$$

where $a$ is some constant.

Next we prove that in the case $D=H$ and $z_{0}=\infty$ the relative capacity and the half-plane capacity coincide.

Theorem 2.6. The capacity relcap $E$ of a bounded set, relatively closed in the half-plane $H$, at the point $z_{0}=\infty$ is equal to the half-plane capacity hcap $E$.

Proof. The expansions (1.1) and (1.2) take the following form after the change of variable $\zeta=-1 / z$ :

$$
\begin{gathered}
\frac{r(\tilde{G}, i \eta)}{2 \eta}=1-2(\operatorname{relcap} E) \eta^{2}+o\left(\eta^{2}\right), \quad \eta \rightarrow 0, \\
\tilde{\phi}(\zeta)=\phi_{G}(-1 / \zeta)=-\operatorname{Im} 1 / \zeta-\operatorname{Im}(\text { hcap } E) \zeta+o(\zeta), \quad \zeta \rightarrow 0,
\end{gathered}
$$

where $\tilde{G}=\{\zeta:-1 / \zeta \in G\}, G=H \backslash E, \zeta=\xi+i \eta$. Consider the harmonic function

$$
u(\zeta)=g_{\tilde{G}}(\zeta, i \eta)-g_{H}(\zeta, i \eta)-2 \eta\left[\tilde{\phi}(\zeta)+\operatorname{Im} \frac{1}{\zeta}\right], \quad \zeta \in \tilde{G} .
$$

At the regular boundary points of the boundary of the domain $\tilde{G}$ we have

$$
\begin{gathered}
u(\zeta)=\log \left|\frac{\zeta-i \eta}{\zeta+i \eta}\right|-2 \eta \operatorname{Im} \frac{1}{\zeta}=\log \left|\frac{1+i z \eta}{1-i z \eta}\right|+2 \eta \operatorname{Im} z \\
=\log \left|(1+i z \eta)\left(1+i z \eta-z^{2} \eta^{2}+o\left(\eta^{2}\right)\right)\right|+2 \eta \operatorname{Im} z \\
=\log \left|1+2 i z \eta-2 z^{2} \eta^{2}+o\left(\eta^{2}\right)\right|+2 \eta \operatorname{Im} z \\
\left.=\log \mid 1-2 y \eta+2\left(y^{2}-x^{2}\right) \eta^{2}+i\left[2 x \eta-4 x y \eta^{2}\right]+o\left(\eta^{2}\right)\right) \mid+2 \eta y \\
=\frac{1}{2} \log \left(1-4 y \eta+8 y^{2} \eta^{2}+o\left(\eta^{2}\right)\right)+2 \eta y=o\left(\eta^{2}\right), \quad \eta \rightarrow 0
\end{gathered}
$$

$(z=x+i y)$. According to the maximum principle we have

$$
u(i \eta)=o\left(\eta^{2}\right), \quad \eta \rightarrow 0 .
$$

This relation together with (2.8) gives

$$
\log \frac{r(\tilde{G}, i \eta)}{r(H, i \eta)}+2(\text { hcap } E) \eta^{2}=o\left(\eta^{2}\right),
$$

which in view of (2.7) gives

$$
\text { relcap } E=\text { hcap } E \text {. }
$$

The theorem is proved. 
We next record some immediate properties of the relative capacity that follow from the properties of the inner radius and the expansion (1.1) (cf. L1]). In what follows the domain $D$ and the point $z_{0}$ are fixed.

Property 1 (Monotonicity). If $E_{1} \subset E_{2}$, then

$$
\text { relcap } E_{1} \leq \text { relcap } E_{2} \text {. }
$$

Proof. The proof is a consequence of the monotonicity of the inner radius

$$
r\left(D \backslash E_{1}, z\right) \geq r\left(D \backslash E_{2}, z\right)
$$

which follows from the inclusion $D \backslash E_{2} \subset D \backslash E_{1}$.

Property 2 (Choquet's inequality). For all $E_{1}, E_{2}$, the inequality

$$
\text { relcap } E_{1}+\operatorname{relcap} E_{2} \geq \operatorname{relcap}\left(E_{1} \cup E_{2}\right)+\operatorname{relcap}\left(E_{1} \cap E_{2}\right)
$$

holds.

Proof. The proof follows from an inequality of Renggli (see $[\mathrm{R}$ ) and the formula (1.1).

Let the domain $D$ be symmetric with respect to the imaginary axis and let $z_{0}$ be a point on this axis, $z_{0} \in \partial D$. For a given set $E \subset D$ we define the set

$$
P E=\left(E \cup E^{*}\right)^{+} \cup\left(E \cap E^{*}\right)^{-},
$$

where $A^{*}$ denotes a set symmetric to $A$ with respect to the imaginary axis, whereas $A^{+}\left(A^{-}\right)$is the intersection of $A$ with the right (left) closed half-plane.

Property 3 (Polarization principle). The following inequality holds:

$$
\text { relcap } E \geq \operatorname{relcap} P E \text {. }
$$

Proof. Consider the set

$$
P_{c}(D \backslash E)=\left((D \backslash E) \cup(D \backslash E)^{*}\right)^{-} \cup\left((D \backslash E) \cap(D \backslash E)^{*}\right)^{+} .
$$

It is easy to see that

$$
P_{c}(D \backslash E)=D \backslash P E .
$$

According to Corollary 1.2 of [D1],

$$
r\left(P_{c}(D \backslash E), z\right) \geq r(D \backslash E, z)
$$

for every point of the imaginary axis contained in $D \backslash E$. It remains just to apply formula (1.1).

Property 4 (Composition principle). Under the hypotheses and notation introduced before Property 3 we have the following inequality:

$$
2 \text { relcap } E \geq \operatorname{relcap}\left(E^{+} \cup\left(E^{+}\right)^{*}\right)+\operatorname{relcap}\left(E^{-} \cup\left(E^{-}\right)^{*}\right) \text {. }
$$

Proof. According to Theorem 1.9 of [D2],

$$
r^{2}(D \backslash E, z) \leq r\left(D \backslash\left(E^{+} \cup\left(E^{+}\right)^{*}\right), z\right) r\left(D \backslash\left(E^{-} \cup\left(E^{-}\right)^{*}\right), z\right)
$$

for the points of the imaginary axis. For both parts of the inequality for $r^{2}(D, z)$ we apply formula (1.1) and arrive at the desired conclusion. 
Property 5. Given a relatively closed subset $E$ of $D$, there exists a sequence of open sets $\left\{B_{n}\right\}_{n=1}^{\infty}$ such that $E \subset B_{n}, n=1,2, \ldots$, and

$$
\lim _{n \rightarrow \infty} \operatorname{relcap}\left(\bar{B}_{n} \cap D\right)=\operatorname{relcap} E \text {. }
$$

Proof. The proof is essentially contained in the proof of a particular case (Lemma 3 of [D2]), noting the representation of the relative capacity in terms of the Green function (see the end of the proof of Theorem 2.1).

Property 6. Suppose that $f$ maps a domain $D$ onto a domain $f(D)$ conformally and univalently such that a boundary point $z_{0}$ is mapped in the sense of boundary correspondence to a point $w_{0} \in \partial f(D)$. We assume further that in a neighborhood of the point $z_{0}$ the boundary $\partial D$ is an analytic arc and the boundary $\partial f(D)$ in a neighborhood of $w_{0}$ is also an analytic arc. If, furthermore, $z_{0}=w_{0}=\infty$ and $\lim _{z \rightarrow \infty} f(z) / z=a$, then

$$
\text { relcap } f(E)=|a|^{2} \text { relcap } E,
$$

and if $z_{0}$ and $w_{0}$ are finite points, then

$$
\text { relcap } f(E)=(\operatorname{relcap} E) /\left|f^{\prime}\left(z_{0}\right)\right|^{2},
$$

for every relatively closed subset $E \subset D, \rho\left(E, z_{0}\right)>0$.

The proof follows from formula (1.1) .

\section{THE BeHAVIOR OF THE RELATIVE CAPACity}

UNDER GEOMETRIC TRANSFORMATIONS OF SUBSETS IN THE DISK

It would be an interesting problem to study the behavior of the relative capacity under simultaneous geometric transformations of the domain $D$ and the set $E \subset D$. However, this problem appears to be very difficult. Therefore we restrict ourselves here only to the case when the domain $D$ is fixed. Furthermore, throughout this section, the domain $D$ is the unit disk $U=U_{z}=\{z:|z|<1\}$, the point $z_{0}=1, E$ is a relatively closed subset of $U$, with $E \not \supset 1$, and relcap $E$ stands for the relative capacity of $E$ with respect to the disk $U$ at the point $z=1$.

We recall the definition of the circular symmetrization of closed and open sets with respect to a given ray (see $[\mathrm{PS}, \mathrm{H}, \mathrm{D} 1])$. Given a real number $a$ let $\gamma_{r}(a)$ be the circle $|z-a|=r$ (for $0<r<\infty$ ), which degenerates to the point $a$ (or $\infty$ ) if $r=0(r=\infty$, resp.). By the circular symmetrization of a closed set $F \subset \overline{\mathbb{C}}$ with respect to the ray $[-\infty, a]$ we mean the transformation of this set onto a symmetric set $\mathrm{Cr}_{a}^{-} F$ which is defined as follows. If, for a given $0 \leq r \leq \infty$, the 'circle' $\gamma_{r}(a)$ does not meet the set $F$, then it has empty intersection with the $\operatorname{set} \mathrm{Cr}_{a}^{-} F$ as well. If $\gamma_{r}(a) \subset F$, then $\gamma_{r}(a) \subset \mathrm{Cr}_{a}^{-} F$. In the remaining cases, the set $\mathrm{Cr}_{a}^{-} F$ intersects $\gamma_{r}(a)$ along a closed arc with center at the ray $[-\infty, a]$, whose linear measure agrees with the measure of the intersection of $F$ with $\gamma_{r}(a)$. It is readily verified that the set $\mathrm{Cr}_{a}^{-} F$ is closed. In the same way we define the result of the circular symmetrization $\mathrm{Cr}_{a}^{+} F$ with respect to the ray $[a, \infty]$. The only difference consists of the fact that the center of the closed arc is now contained on the ray $[a, \infty]$. In the same way, we define the circular symmetrizations $\mathrm{Cr}_{a}^{+}, \mathrm{Cr}_{a}^{-}$of open sets, now taking an open arc in place of a closed arc. 
Theorem 3.1. The following inequalities hold:

$$
\text { relcap } E \geq \operatorname{relcap}\left[\left(\mathrm{Cr}_{o}^{-\bar{E}}\right) \cap U\right],
$$

$$
\text { relcap } E \geq \operatorname{relcap}\left[\left(\mathrm{Cr}_{a}^{-}(E \cup \Sigma)\right) \backslash \Sigma\right], \quad(a \leq 0),
$$$$
\text { relcap } E \geq \operatorname{relcap}\left[\left(\mathrm{Cr}_{a}^{+}(E \cup \Sigma)\right) \backslash \Sigma\right], \quad(a \geq 1),
$$

where $\Sigma=\{z:|z| \geq 1\}$.

Proof. All three inequalities can be proved in a unified way, applying formula (1.1) and Polya's result on the behavior of the inner radius of a domain under circular symmetrization (see [H, D1] ). For instance, from Polya's theorem it follows that for every $x, 0<x<1$,

$$
r(U \backslash E, x) \leq r\left(\mathrm{Cr}_{o}^{+}(U \backslash E), x\right)=r\left(U \backslash\left[\mathrm{Cr}_{o}^{-}(\bar{E}) \cap U\right], x\right) .
$$

It remains to apply formula (1.1) with $D=U$. Analogously, for $a \leq 0$

$$
r(U \backslash E, x) \leq r\left(\mathrm{Cr}_{a}^{+}(U \backslash E), x\right)=r\left(U \backslash\left[\mathrm{Cr}_{a}^{-}(E \cup \Sigma) \backslash \Sigma\right], x\right),
$$

and for $a \geq 1$ we have

$$
r(U \backslash E, x) \leq r\left(\mathrm{Cr}_{a}^{-}(U \backslash E), x\right)=r\left(U \backslash\left[\mathrm{Cr}_{a}^{+}(E \cup \Sigma) \backslash \Sigma\right], x\right) .
$$

The theorem is proved.

Figure 1 provides an illustration of a set $E$ and Figures 2, 3, and 4 illustrate its deformation under each of the transformations in the inequalities (3.2), and (3.3), (3.4), respectively.



Figure 1. The closed set $E$.

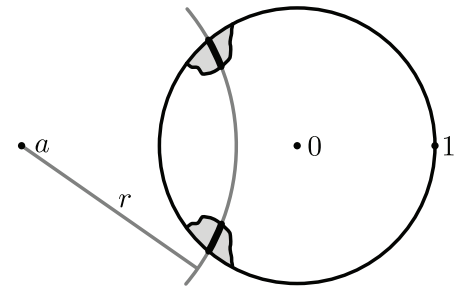

Figure 3

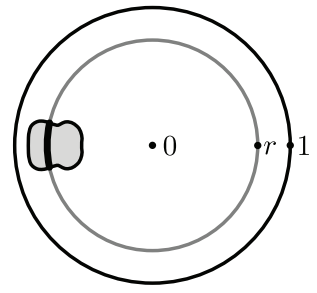

Figure 2. Circular symmetrization.

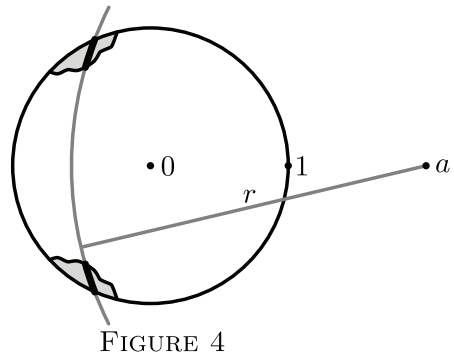

Figure 4

Circular symmetrization. 
The Steiner symmetrization of an open set $B$ with respect to the real axis is defined by

$$
\text { St } B=\{x+i y: B \cap \lambda(x) \neq \emptyset, 2|y|<\mu(B \cap \lambda(x))\},
$$

where $\lambda(x)$ is the line $\operatorname{Re} z=x$ and $\mu(\cdot)$ is the linear Lebesgue measure.

Theorem 3.5. The following inequality holds:

$$
\operatorname{relcap} E \geq \operatorname{relcap}[U \backslash \operatorname{St}(U \backslash E)] \text {. }
$$

Proof. In the same way as in the proof of Theorem 3.1 it is enough to apply a theorem of Polya and Szegö [PS],

$$
r(U \backslash E, x) \leq r(\operatorname{St}(U \backslash E), x),
$$

and to note the equality

$$
U \backslash[U \backslash \operatorname{St}(U \backslash E)]=\operatorname{St}(U \backslash E) .
$$

The proof is complete.



FIGURE 5. Steiner symmetrization.

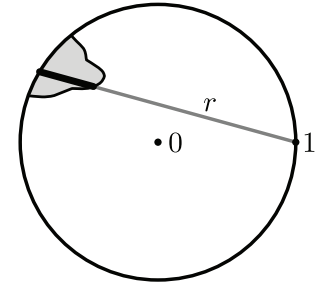

Figure 6. Radial transformation.

We consider the following radial Marcus transformation (see [M1]), which transforms an open set $B, z_{0} \in B$ onto the starlike set

$$
\mathrm{M}_{z_{o}} B=\left\{z_{0}+r e^{i \theta}: 0 \leq r<M(\theta, B), 0 \leq \theta \leq 2 \pi\right\},
$$

where

$$
M(\theta, B)=\rho \exp \left(\int_{F(\rho, \theta, B)} \frac{d r}{r}\right), \quad F(\rho, \theta, B)=\left\{r: z_{0}+r e^{i \theta} \in B, \rho \leq r<\infty\right\} .
$$

Here $M(\theta, B)$ is independent of $\rho$ and $\left\{z:\left|z-z_{0}\right| \leq \rho\right\} \subset B$. For open sets $B$, lying in the disk $U$ and containing a relative neighborhood $\{z:|z-1|<\varepsilon\} \cap U$ for some $\varepsilon>0$ we define $\mathrm{M} B \equiv \mathrm{M}_{1} B$ as above, but with a constraint on $\theta: \pi / 2<\theta<3 \pi / 2$.

Theorem 3.8. The following inequality holds:

$$
\text { relcap } E \geq \operatorname{relcap}[U \backslash \mathrm{M}(U \backslash E)] \text {. }
$$

Proof. Let $\left\{B_{n}\right\}_{n=1}^{\infty}$ be a sequence from Property 5 for the given set $E, D=U$, and let $E_{n}=\bar{B}_{n} \cap U, n=1,2, \ldots$ According to a theorem of Marcus [M1] for a fixed integer $n$ and for values of $x$ sufficiently close to $1,0<x<1$, we have

$$
r\left(U \backslash E_{n}, x\right) \leq r\left(\mathrm{M}_{x}\left(U \backslash E_{n}\right), x\right)=r\left(U \backslash\left[U \backslash \mathrm{M}_{x}\left(U \backslash E_{n}\right)\right], x\right) .
$$

On the other hand, because of the openness of $B_{n}$, one can take $x$ so close to 1 that

$$
\mathrm{M}_{x}\left(U \backslash E_{n}\right) \subset \mathrm{M}(U \backslash E) .
$$


Therefore

$$
r\left(U \backslash\left[U \backslash \mathrm{M}_{x}\left(U \backslash E_{n}\right)\right], x\right) \leq r(U \backslash[U \backslash \mathrm{M}(U \backslash E)], x) .
$$

It remains to apply formula (1.1) and Property 5. The proof is complete.

Let $A=\left\{\alpha_{k}\right\}_{k=1}^{n}$ be a family of positive numbers with $\sum_{k=1}^{n} \alpha_{k}=1$. We consider an averaging transformation (see [M2]) which assigns the starlike set

$$
\mathbb{R}_{A}^{z_{0}}\left\{B_{k}\right\}_{k=1}^{n}=\left\{z_{0}+r e^{i \theta}: 0 \leq r<\Pi_{k=1}^{n}\left(M\left(\theta, B_{k}\right)\right)^{\alpha_{k}}, 0 \leq \theta \leq 2 \pi\right\}
$$

to a family of open subsets $B_{k}, k=1, \ldots, n$, containing a point $z_{0}$; here $M(\theta, B)$ is given above. By Theorem 2.2 of [M2],

$$
\Pi_{k=1}^{n}\left(r\left(B_{k}, z_{0}\right)\right)^{\alpha_{k}} \leq r\left(\mathbb{R}_{A}^{z_{0}}\left\{B_{k}\right\}_{k=1}^{n}, z_{0}\right) .
$$

For a family of open subsets of the disk containing the neighborhood $\{z:|z-1|<$ $\varepsilon\} \cap D$ for some $\varepsilon>0$, the set

$$
\mathbb{R}_{A}\left\{B_{k}\right\}_{k=1}^{n} \equiv \mathbb{R}_{A}^{1}\left\{B_{k}\right\}_{k=1}^{n}
$$

is defined but under a constraint on $\theta: \pi / 2<\theta<3 \pi / 2$. Repeating the proof of the preceding theorem and replacing $\mathrm{M}_{x}$ with $\mathbb{R}_{A}^{x}$ and applying Marcus' theorem and Property 5 , we arrive at the following result.

Theorem 3.10. For every collection of sets $\left\{E_{k}\right\}_{k=1}^{n}$ and for every family $A=$ $\left\{\alpha_{k}\right\}_{k=1}^{n}$ of positive numbers with $\sum_{k=1}^{n} \alpha_{k}=1$, the following inequality holds:

$$
\sum_{k=1}^{n} \alpha_{k} \text { relcap } E_{k} \geq \operatorname{relcap}\left[U \backslash \mathbb{R}_{A}\left\{U \backslash E_{k}\right\}_{k=1}^{n}\right] \text {. }
$$

Applying Theorem 3.10 to the case when the collection of sets consists of two sets $\{E,\{z: \bar{z} \in E\}\}$ and the family of numbers $A=\left\{\frac{1}{2}, \frac{1}{2}\right\}$, we obtain the following theorem.

Theorem 3.11. The following inequality holds:

relcap $E \geq \operatorname{relcap} \mathrm{R} E$,

$\mathrm{R} E=\left\{1+r e^{i \theta}:(M(\theta, U \backslash E) M(2 \pi-\theta, U \backslash E))^{1 / 2} \leq r<-2 \cos \theta, \pi / 2<\theta<3 \pi / 2\right\}$.

\section{Applichtions to Bounded holomorphic FunCtions in the DisK}

The important role of the Schwarzian derivative in geometric function theory is well-known. In particular, it is interesting to find geometric estimates of the Schwarzian derivative. In this section are given estimates of the Schwarzian derivative in terms of the geometry of the image domain of the unit disk under a bounded holomorphic function. These estimates follow in a unified way from the connection of the Schwarzian derivative and the relative capacity at a boundary point. Note that distortion estimates involving the Schwarzian derivative originate, in particular, from generalized rigidity theorems. For instance, Theorem 2.6 of [TV] can now be understood as a distortion result under the hypothesis that the image of the disk lies within a horocycle. In this context, there also occurs a natural condition for the initial coefficients of the expansion of the function in the neighborhood of a boundary point (see also [S, Theorem 4], [D3, Proposition 2]). Here a more general situation is considered, that being when the image of the disk has a property, characteristic for the application of the symmetrization method $[\mathrm{H}]$. In accordance 
with what was said above, we denote by $\mathcal{B}$ the class of functions $f$ holomorphic in the disk $U_{z}=\{z:|z|<1\}, f\left(U_{z}\right) \subset U_{w}$ with the asymptotic expansion

$$
f(z)=1+a_{1}(z-1)+a_{2}(z-1)^{2}+a_{3}(z-1)^{3}+\angle o\left((z-1)^{3}\right),
$$

where $a_{1}>0, \operatorname{Re}\left(2 a_{2}+a_{1}\left(1-a_{1}\right)\right)=0$ and $\angle o\left((z-1)^{3}\right)$ is an infinitesimal quantity in comparison with $(z-1)^{3}$ as $z \rightarrow 1$ in any Stolz angle in $U_{z}$ with vertex at $z=1$. The condition for the real part of the combination of initial coefficients of the function in the definition of class $\mathcal{B}$ is a necessary normalization for obtaining estimates for the Schwarzian at a boundary point of the unit disk. The corresponding condition for a coefficient of the holomorphic functions in the upper half-plane has the form $\operatorname{Im} c_{2}=0$. The geometric meaning of this condition is given in [D3].

In the rest of this section relcap $E$ stands for the relative capacity of $E$ with respect to the disk $U$ at the point $z=1$.

Theorem 4.1. For every function $f$ of class $\mathcal{B}$ and for every set $E$, closed with respect to the disk $U_{w}$ and lying in the complement $U_{w} \backslash f\left(U_{z}\right)$ at a positive distance from the point $z=1$, the following inequality for the Schwarzian derivative holds:

$$
-\frac{1}{6} \operatorname{Re} S_{f}(1):=-\operatorname{Re}\left(\frac{a_{3}}{a_{1}}-\frac{a_{2}^{2}}{a_{1}^{2}}\right) \geq a_{1}^{2} \text { relcap } E .
$$

The equality holds for functions $f$ of class $\mathcal{B}$, mapping conformally and univalently the disk $U_{z}$ onto the domain $U_{w} \backslash E$.

Proof. In view of the monotonicity of the inner radius and formula (1.1) we have for points $x$ on the segment $(0,1)$,

$$
\begin{aligned}
& \frac{r\left(f\left(U_{z}\right), f(x)\right)}{r\left(U_{w}, f(x)\right)} \leq \frac{r\left(U_{w} \backslash E, f(x)\right)}{r\left(U_{w}, f(x)\right)}=1-2(\text { relcap } E)|f(x)-1|^{2}+o\left(|f(x)-1|^{2}\right) \\
& =1-2 a_{1}^{2}(\operatorname{relcap} E)(1-x)^{2}+o\left((x-1)^{2}\right), \quad x \rightarrow 1 .
\end{aligned}
$$

In the case $f\left(U_{z}\right)=U_{w} \backslash E$, the equality holds in (4.2). On the other hand, from the result of Hayman [ $\mathrm{H}$, Theorem 4.7] it follows that

$$
\frac{r\left(f\left(U_{z}\right), f(x)\right)}{r\left(U_{w}, f(x)\right)} \geq \frac{\left|f^{\prime}(x)\right| r\left(U_{z}, x\right)}{r\left(U_{w}, f(x)\right)}=\frac{\left|f^{\prime}(x)\right|\left(1-x^{2}\right)}{1-|f(x)|^{2}},
$$

where, furthermore, the sign of equality holds for univalent functions. We next find the asymptotic behavior of the right hand side of (4.3):

$$
\begin{gathered}
\frac{\left|f^{\prime}(x)\right|\left(1-x^{2}\right)}{1-|f(x)|^{2}} \\
=\frac{\left|a_{1}-2 a_{2}(1-x)+3 a_{3}(1-x)^{2}+o\left((1-x)^{3}\right)\right|(1-x)(2-(1-x))}{1-\left(1-a_{1}(1-x) !+\operatorname{Re} a_{2}(1-x)^{2}-\operatorname{Re} a_{3}(1-x)^{3}+o\left((1-x)^{3}\right)\right)^{2}+o\left((1-x)^{3}\right)} \\
=\frac{\left|2 a_{1}+(1-x)\left(-4 a_{2}-a_{1}\right)+(1-x)^{2}\left(6 a_{3}+2 a_{2}\right)+o\left((1-x)^{2}\right)\right|}{2 a_{1}+(1-x)\left(-a_{2}^{2}-2 \operatorname{Re} a_{2}\right)+(1-x)^{2}\left(2 \operatorname{Re} a_{3}+2 a_{1} \operatorname{Re} a_{2}\right)+o\left((1-x)^{2}\right)} \\
=\mid 1+(1-x)(-U+V)+(1-x)^{2}\left[\frac{3 a_{3}}{a_{1}}+\frac{a_{2}}{a_{1}}-\frac{\operatorname{Re} a_{3}}{a_{1}}-\operatorname{Re} a_{2}-U V+V^{2}\right] \\
+o\left((1-x)^{2}\right) \mid \equiv W,
\end{gathered}
$$


where $U=\frac{2 a_{2}}{a_{1}}+\frac{1}{2}, V=\frac{a_{1}}{2}+\frac{\operatorname{Re} a_{2}}{a_{1}}$. Next, writing

$$
Y \equiv \frac{3 a_{3}}{a_{1}}+\frac{a_{2}}{a_{1}}-\frac{\operatorname{Re} a_{3}}{a_{1}}-a_{2}-\frac{2 a_{2} \operatorname{Re} a_{2}}{a_{1}^{2}}-\frac{a_{1}}{4}-\frac{\operatorname{Re} a_{2}}{2 a_{1}}+\frac{a_{1}^{2}}{4}+\frac{\left(\operatorname{Re} a_{2}\right)^{2}}{a_{1}^{2}}
$$

we have

$$
\begin{gathered}
W=\left|1+(1-x)\left[\frac{-\operatorname{Re} a_{2}+a_{1}^{2}-a_{1}}{2 a_{1}}-i \frac{2 \operatorname{Im} a_{2}}{a_{1}}\right]+Y(1-x)^{2}+o\left((1-x)^{2}\right)\right| \\
=\left\{\left[1+(1-x)^{2} \operatorname{Re} Y+o\left((1-x)^{2}\right)\right]^{2}+(1-x)^{2} \frac{4\left(\operatorname{Im} a_{2}\right)^{2}}{a_{1}^{2}}+o\left((1-x)^{2}\right)\right\}^{1 / 2} \\
=1+(1-x)^{2}\left(\frac{2 \operatorname{Re} a_{3}}{a_{1}}-\frac{2\left(\operatorname{Re} a_{2}\right)^{2}}{a_{1}^{2}}+\frac{2\left(\operatorname{Im} a_{2}\right)^{2}}{a_{1}^{2}}\right)+o\left((1-x)^{2}\right) \\
=1-2 \operatorname{Re}\left(-\frac{a_{3}}{a_{1}}+\frac{a_{2}^{2}}{a_{1}^{2}}\right)(1-x)^{2}+o\left((1-x)^{2}\right), \quad x \rightarrow 1 .
\end{gathered}
$$

Substituting this resulting asymptotic expansion in (4.3) and adding (4.3) and (4.2), we arrive at the required inequality. The theorem is proved.

Remark 4.4. It can be proved that the condition

$$
\operatorname{Re}\left(2 a_{2}+a_{1}\left(1-a_{1}\right)\right)=0
$$

is essential for Theorem 4.1] (cf. [D3, p. 651]).

Remark 4.5. It is well-known that functions of class $\mathcal{B}$ satisfy $\operatorname{Im} S_{f}(1)=0$ (see $\underline{\mathrm{S}}$, TV]). Therefore in the inequality of Theorem 4.1 and in the following applications, the real part of the Schwarzian could be replaced with $S_{f}(1)$.

Some applications of Theorem 4.1 to the proof of geometric properties of holomorphic functions are given, for instance, in the following statements (cf. [S, TV, LLN, D3]).

Theorem 4.6. Let the function $f$ be of class $\mathcal{B}$, and let the angular Lebesgue measure of the intersection of the image of the unit disk $f\left(U_{z}\right)$ with every circle $|w|=r, 0<r<1$, be smaller than or equal to $\alpha, 0<\alpha<2 \pi$. Then

$$
\operatorname{Re} \frac{S_{f}(1)}{\left(f^{\prime}(1)\right)^{2}} \leq-\frac{3 \pi^{2}}{2 \alpha^{2}}\left[\left(\frac{\alpha}{\pi}-1\right)^{2}+1\right] .
$$

The case of equality holds for the function

$$
f_{\alpha}(z)=\left(\frac{z-1+\sqrt{2 z^{2}+2}}{z+1}\right)^{\alpha / \pi},
$$

which maps the unit disk $U_{z}$ conformally and univalently onto the sector $B(\alpha)=$ $\{z:|z|<1,|\arg z|<\alpha / 2\}$.

Proof. By Theorems 4.1 and 3.1 (inequality (3.2) ) we have

$$
-\frac{1}{6} \operatorname{Re} \frac{S_{f}(1)}{\left(f^{\prime}(1)\right)^{2}} \geq \operatorname{relcap} E \geq \operatorname{relcap}\left[\left(\mathrm{Cr}_{o}^{-} \bar{E}\right) \cap U_{w}\right]
$$

for every set $E$ relatively closed with respect to $U_{w}$, lying in $U_{w} \backslash f\left(U_{z}\right)$ and having a positive distance from the point $z=1$. Set $E=U_{w} \backslash f\left(U_{z}\right)$. If the distance from $U_{w} \backslash f\left(U_{z}\right)$ to the point $z=1$ is zero, then we set

$$
E=\left(U_{w} \backslash f\left(U_{z}\right)\right) \backslash\{z:|z-1|<\varepsilon\}
$$


for a sufficiently small $\varepsilon>0$. If $\beta$ is a number with $0<\alpha<\beta<2 \pi$, then by the hypothesis of the theorem, we can choose $\varepsilon$ such that the set $\mathrm{Cr}_{o}^{-} \bar{E}$ contains $U_{w} \backslash B(\beta)$. From the monotonicity of the capacity and again by Theorem 4.1 we obtain

$$
\operatorname{relcap}\left[\left(\mathrm{Cr}_{o}^{-} \bar{E}\right) \cap U_{w}\right] \geq \operatorname{relcap}\left[U_{w} \backslash B(\beta)\right]=-\frac{1}{6} \operatorname{Re} \frac{S_{f_{\beta}}(1)}{\left(f_{\beta}^{\prime}(1)\right)^{2}} .
$$

The proof now follows by comparing the two obtained inequalities, computing the derivative of the function $f_{\beta}$ and letting $\beta \rightarrow \alpha$.

Theorem 4.8. If a univalent function $f$ of class $\mathcal{B}$ does not take in the disk $U_{z}$ a value $w_{0} \in U_{w}$, then it satisfies the inequality (4.9),

$$
\operatorname{Re} \frac{S_{f}(1)}{\left(f^{\prime}(1)\right)^{2}} \leq-\frac{3}{4}\left(\frac{1-\rho}{1+\rho}\right)^{2},
$$

where $\rho=\left|w_{o}\right|$. Here equality holds, for instance, for the function of Pick, $w=$ $f(z ; \rho)$ given for $\rho \neq 0$ by the equation

$$
\frac{4 \rho z}{(1+\rho)^{2}(1-z)^{2}}=\frac{w}{(1-w)^{2}} .
$$

This function maps the unit disk $U_{z}$ conformally and univalently onto the unit disk $U_{w}$ with the segment $[-1,-\rho]$ removed.

Proof. The function $g(z)=f(f(z) ; \beta)$ is in the class $\mathcal{B}$ for a fixed $\beta, 0<\beta<1$. Therefore it satisfies inequality (4.7) from the proof of the previous theorem with $f$ replaced with $g$. For values of $\beta$ close to one, the image of the disk $U_{z}$ under $g$ does not contain any of the circles $\{w:|w|=r\}, \rho(\beta)<r<1$, where $\lim _{\beta \rightarrow 1} \rho(\beta)=\rho$. Choosing $\varepsilon$ from the previous proof to be smaller than $1-\beta$, we conclude that the set $\mathrm{Cr}_{o}^{-} \bar{E}$ contains the segment $[-1,-\rho(\beta)]$. From the monotonicity of the capacity and Theorem 4.1 we obtain

$$
\operatorname{relcap}\left[\left(\mathrm{Cr}_{o}^{-} \bar{E}\right) \cap U_{w}\right] \geq \operatorname{relcap}(-1,-\rho(\beta)]=-\frac{1}{6} \operatorname{Re} \frac{S_{h}(1)}{\left(h^{\prime}(1)\right)^{2}},
$$

where $h(z)=f(z ; \rho(\beta))$. Comparison with (4.7) $(f=g)$ gives

$$
\operatorname{Re} \frac{S_{g}(1)}{\left(g^{\prime}(1)\right)^{2}} \leq \operatorname{Re} \frac{S_{h}(1)}{\left(h^{\prime}(1)\right)^{2}} .
$$

Letting $\beta \rightarrow 1$ and computing the derivative of the function $f(z ; \rho)$ we arrive at the stated inequality. The proof is complete.

Theorem 4.10. Let the function $f$ be of class $\mathcal{B}$ and suppose that the linear Lebesgue measure of the intersection of the image $f\left(U_{z}\right)$ with the imaginary axis is at most $2 t, 0<t<1$. Then the following inequality holds:

$$
\operatorname{Re} \frac{S_{f}(1)}{\left(f^{\prime}(1)\right)^{2}} \leq-\frac{3}{2}\left(\frac{1-t^{2}}{1+t^{2}}\right)^{2} .
$$

The equality holds, for instance, for the function $\tilde{f}_{t}(z)$, given by the equation

$$
\frac{2 t}{1+t^{2}} \frac{z}{1-z^{2}}=\frac{w}{1-w^{2}} .
$$

This function maps the unit disk $U_{z}$ conformally and univalently onto the disk $U_{w}$ with the segments $[ \pm i t, \pm i]$ removed. 
Proof. According to Theorems 4.1 and 3.5 we have

$$
-\frac{1}{6} \operatorname{Re} \frac{S_{f}(1)}{\left(f^{\prime}(1)\right)^{2}} \geq \operatorname{relcap} E \geq \operatorname{relcap}[U \backslash \operatorname{St}(U \backslash E)]
$$

for every set $E$ relatively closed with respect to $U_{w}$ lying in the set $U_{w} \backslash f\left(U_{z}\right)$ and having a positive distance to the point $z=1$. We replace the set $E$ with the intersection of $U_{w} \backslash f\left(U_{z}\right)$ with the imaginary axis. Then from the monotonicity of the capacity and again by Theorem 4.1 it follows that

$$
\operatorname{relcap}[U \backslash \operatorname{St}(U \backslash E)] \geq \operatorname{relcap}\left[U_{w} \backslash \tilde{f}_{t}\left(U_{z}\right)\right]=-\frac{1}{6} \operatorname{Re} \frac{S_{\tilde{f}_{t}}(1)}{\left(\tilde{f}_{t}^{\prime}(1)\right)^{2}} .
$$

It only remains to compute the derivative on the right hand side, which is an easy exercise. The theorem is proved.

\section{ACKNOWLEDGEMENTS}

The authors are indebted to the referees for their valuable comments. This work was completed during the visit of the first author to the University of Turku, Finland.

\section{REFERENCES}

[A] Lars Valerian Ahlfors, Collected papers. Vol. 1. 1929-1955, edited with the assistance of Rae Michael Shortt. Contemporary Mathematicians, Birkhäuser Boston, Mass., 1982. MR688648(84k:01066a)

[AB] Lars Ahlfors and Arne Beurling, Conformal invariants and function-theoretic null-sets, Acta Math. 83 (1950), 101-129. MR0036841(12,171c)

[D1] V. N. Dubinin, Symmetrization in the geometric theory of functions of a complex variable (Russian), Uspekhi Mat. Nauk 49 (1994), no. 1(295), 3-76, DOI 10.1070/RM1994v049n01ABEH002002; English transl., Russian Math. Surveys 49 (1994), no. 1, 1-79. MR1307130 (96b:30054)

[D2] V. N. Dubinin, Lower bounds for the half-plane capacity of compact sets and symmetrization (Russian, with Russian summary), Mat. Sb. 201 (2010), no. 11, 77-88, DOI 10.1070/SM2010v201n11ABEH004125; English transl., Sb. Math. 201 (2010), no. 11-12, 1635-1646. MR2768554 (2011m:30059)

[D3] V. N. Dubinin, On the boundary values of the Schwarzian derivative of a regular function (Russian, with Russian summary), Mat. Sb. 202 (2011), no. 5, 29-44, DOI 10.1070/SM2011v202n05ABEH004159; English transl., Sb. Math. 202 (2011), no. 5-6, 649663. MR 2841516 (2012f:30047)

[H] W. K. Hayman, Multivalent functions, 2nd ed., Cambridge Tracts in Mathematics, vol. 110, Cambridge University Press, Cambridge, 1994. MR.1310776 (96f:30003)

[He] Joseph Hersch, On the reflection principle and some elementary ratios of conformal radii, J. Analyse Math. 44 (1984/85), 251-268, DOI 10.1007/BF02790200. MR801297 (87h:30020)

[LLN] Steven Lalley, Gregory Lawler, and Hariharan Narayanan, Geometric interpretation of halfplane capacity, Electron. Commun. Probab. 14 (2009), 566-571, DOI 10.1214/ECP.v141517. MR2576752 (2011b:60332)

[L1] Gregory F. Lawler, Conformally invariant processes in the plane, Mathematical Surveys and Monographs, vol. 114, American Mathematical Society, Providence, RI, 2005. MR2129588 (2006i:60003)

[L2] G. Lawler, Schramm-Loewner evolution (SLE), Statistical mechanics, IAS/Park City Math. Ser., vol. 16, Amer. Math. Soc., Providence, RI, 2009, pp. 231-295. MR2523461 (2011d:60244)

[L3] Gregory F. Lawler, Conformal invariance and 2D statistical physics, Bull. Amer. Math. Soc. (N.S.) 46 (2009), no. 1, 35-54, DOI 10.1090/S0273-0979-08-01229-9. MR2457071 (2010a:60283) 
[M1] Moshe Marcus, Transformations of domains in the plane and applications in the theory of functions, Pacific J. Math. 14 (1964), 613-626. MR0165093 (29 \#2382)

[M2] Moshe Marcus, Radial averaging of domains, estimates for Dirichlet integrals and applications, J. Analyse Math. 27 (1974), 47-78. MR0477029 (57 \#16573)

[O] M. Ohtsuka, Dirichlet problem, extremal length, and prime ends, Van Nostrand Reinhold, 1970.

[PS] G. Pólya and G. Szegö, Isoperimetric Inequalities in Mathematical Physics, Annals of Mathematics Studies, no. 27, Princeton University Press, Princeton, N. J., 1951. MR.0043486(13,270d)

[Pom] Ch. Pommerenke, Boundary behaviour of conformal maps, Grundlehren der Mathematischen Wissenschaften [Fundamental Principles of Mathematical Sciences], vol. 299, SpringerVerlag, Berlin, 1992. MR,1217706 (95b:30008)

[R] Heinz Renggli, An inequality for logarithmic capacities, Pacific J. Math. 11 (1961), 313314. MR0155992 (27 \#5925)

[RW] S. Rohde and C. Wong, Half-plane capacity and conformal radius, Proc. Amer. Math. Soc. 142 (2014), no. 3, 931-938. MR3148527

[S] David Shoikhet, Another look at the Burns-Krantz theorem, J. Anal. Math. 105 (2008), 19-42, DOI 10.1007/s11854-008-0030-8. MR2438420(2009h:30040)

[TV] Roberto Tauraso and Fabio Vlacci, Rigidity at the boundary for holomorphic self-maps of the unit disk, Complex Variables Theory Appl. 45 (2001), no. 2, 151-165. MR.1909431 (2003e:30039)

Far Eastern Federal University, Vladivostok, Russia

Current address: Institute of Applied Mathematics, Vladivostok, Russia

E-mail address: dubinin@iam.dvo.ru

Department of Mathematics and Statistics, University of Turku, Turku 20014, FINLAND

E-mail address: vuorinen@utu.fi 